\title{
Urinary extracellular vesicles: a rising star in bladder cancer management
}

\author{
Fumihiko Urabe $^{1,2} \wedge$, Takahiro Kimura ${ }^{1}$, Kagenori Ito ${ }^{1,2}$, Yusuke Yamamoto ${ }^{2}$, Shunsuke Tsuzuki $^{1}$, \\ Jun Miki ${ }^{1,3}$, Takahiro Ochiya ${ }^{4}$, Shin Egawa ${ }^{1}$ \\ ${ }^{1}$ Department of Urology, The Jikei University School of Medicine, Tokyo, Japan; ${ }^{2}$ Division of Cellular Signaling, National Cancer Center Research \\ Institute, Tokyo, Japan; ${ }^{3}$ Department of Urology, The Jikei University Kashiwa Hospital. Chiba, Japan; ${ }^{4}$ Department of Molecular and Cellular \\ Medicine, Tokyo Medical University, Tokyo, Japan \\ Contributions: (I) Conception and design: F Urabe, T Kimura, K Ito; (II) Administrative support: F Urabe; (III) Provision of study materials or \\ patients: F Urabe; (IV) Collection and assembly of data: F Urabe, S Tsuzuki; (V) Data analysis and interpretation: F Urabe, Y Yamamoto; (VI) \\ Manuscript writing: All authors; (VII) Final approval of manuscript: All authors. \\ Correspondence to: Takahiro Kimura, MD, PhD. The Jikei University School of Medicine, 3-25-8, Nishi-Shimbashi Minato-ku, Tokyo, 105-8461, \\ Japan. Email: tkimura@jikei.ac.jp.
}

\begin{abstract}
Clinically, the detection of bladder cancer (BCa) typically requires cystoscopy, which is potentially harmful and sometimes accompanied by adverse effects. Thus, new biomarkers are desirable for improving the management of BCa. Recently, "liquid biopsy" has received enormous attentions and has been extensively studied due to its promising clinical implication for precise medicine. Especially, extracellular vesicles (EVs) have attracted strong interest as a potential source of biomarkers. EVs have been reported to be found in almost all types of body fluids and are easy to collect. In addition, EVs tightly reflect the current state of the disease by inheriting specific biomolecules from their parental cells. Urinary EVs have gained great scientific interest in the field of BCa biomarker research since urine is in direct contact with $\mathrm{BCa}$ and can contain large amounts of EVs from the tumour microenvironment. To date, various kinds of biomolecules, including noncoding RNAs, mRNAs, and proteins, have been investigated as biomarkers in urinary EVs. In this narrative review, we summarize the recent advances regarding urinary EVs as noninvasive biomarkers in patients with BCa. The current hurdles in the clinical implications of EV-based liquid biopsy and the potential applications of EV research are also discussed.
\end{abstract}

Keywords: Extracellular vesicles (EVs); bladder cancer (BCa); biomarker; exosome

Submitted Jun 30, 2020. Accepted for publication Oct 19, 2020.

doi: $10.21037 /$ tau-20-1039

View this article at: http://dx.doi.org/10.21037/tau-20-1039

\section{Introduction}

Bladder cancer (BCa) is the $10^{\text {th }}$ most commonly diagnosed cancer type in the world; it ranks $13^{\text {th }}$ in Japan and $6^{\text {th }}$ in the USA. It occurs approximately four times more frequently in men than in women; therefore, $\mathrm{BCa}$ in men is the $8^{\text {th }}$ most commonly diagnosed cancer in Japan and the $4^{\text {th }}$ most commonly diagnosed cancer in the USA, whereas in women, it ranks $16^{\text {th }}$ in Japan and $11^{\text {th }}$ in the USA (1-3).

Current diagnostic tools for detecting BCa are cytology and cystoscopy. Cytology of the voided bladder urine specimen is a common and non-invasive screening test for $\mathrm{BCa}$. Although cytology is very specific (approximately $80 \%$ ), its overall low sensitivity (approximately $40 \%$ ) is a

^ ORCID: 0000-0002-2599-8183. 
clear limitation. Indeed, it has a relatively high sensitivity for high-grade or advanced $\mathrm{BCa}$, whereas it has a low sensitivity for low-grade or early-stage $\mathrm{BCa}$, ranging only $5 \%$ to $30 \%$ (4-7). Therefore, a positive cytology indicates the presence of $\mathrm{BCa}$; however, negative cytology cannot exclude the presence of BCa. Cystoscopy is the gold standard for cancer diagnosis; however, its effectiveness is operator-dependent, and it is highly invasive and can cause complications, such as frequent urination, pain during urination, visible haematuria, and urinary infection $(8,9)$. In addition, it is sometimes difficult to identify carcinoma in situ (CIS) by cystoscopy. Recently, new biomarker-based urine tests were approved by the Food and Drug Administration (FDA). Three protein-based noninvasive urine tests [bladder tumour antigen (BTA), nuclear matrix protein 22 (NMP22), and ImmunoCyt/uCyt], which are approved by the FDA and commercially available, are not recommended in the current guidelines because of heterogeneous data (10). In addition, UroVysion, which is a fluorescence in situ hybridization (FISH) probe set to detect $\mathrm{BCa}$, is limited by low sensitivity for low-grade BCa (11). Thus, UroVysion has not been approved for the initial detection of $\mathrm{BCa}$ but for assessing responses to intravesical Bacillus CalmetteGuerin (BCG) therapy (12). To date, many efforts have been made to establish effective molecular markers for BCa. However, these urine tests are currently not used in daily clinical practice. Further research is required to establish more clinically useful tests, especially to detect low-grade and early-stage BCa.

In the search for a novel biomarker, a number of studies have focused on extracellular vesicles (EVs). EVs can be detected in almost all kinds of body fluids, and as we introduce later, EVs contain a complex mixture of RNAs, DNAs, and proteins that are covered by or localized on a lipid bilayer membrane (13). In this review, we summarize the current knowledge of EVs as biomarkers for $\mathrm{BCa}$ and discuss the potential of EVs in BCa management.

\section{Classification of EVs}

EVs comprise various types of small vesicles, ranging from approximately 50 to $4,000 \mathrm{~nm}$ in size, which are secreted by almost all kinds of cells into the extracellular environment (14). Based on the size and secretion mechanism, EVs are often classified into three main classes: exosomes, microvesicles, and apoptotic bodies (15). Exosomes are nanosized bilayer EVs with diameters of approximately 50 to $100 \mathrm{~nm}$. Exosomes are first generated by a process of inward and reverse budding of an endosomal membrane of multivesicular bodies and are consequently secreted into the extracellular environment $(16,17)$. Microvesicles, which are larger than exosomes (100$1,000 \mathrm{~nm}$ ), are directly released from cellular membranes by budding or shedding (18). Apoptotic bodies vary in size $(50-4,000 \mathrm{~nm})$ and are released from the cellular membrane during programmed cell death (19). Although the origins of these vesicles are distinct, their characteristics, such as size and density, are similar, and it is impossible to completely separate these vesicles with the currently available technologies. Thus, as the International Society for Extracellular Vesicles (ISEV) has recommended (20), we use EVs as a collective term covering all types of EVs.

Accumulating evidence has shown that EVs are key mediators of cell-to-cell communication through transferring their components, including miRNAs, mRNAs, DNAs, and proteins (13). Especially in the cancer research field, accumulated studies have helped us gain a deeper understanding of the mechanism of tumour progression (21). The role of EVs in tumour progression or metastasis, which is directly related to patient mortality, has repeatedly been reported. In focal tumour progression and systemic cancer metastasis, EVs secreted from cancer cells as well as other cells, such as tumour endothelial cells (TECs), cancerassociated fibroblasts (CAFs), and tumour-educated platelets (TEPs), are reported to have distinct roles (22). Therefore, it is valuable to elucidate how cancer cells educate and communicate with surrounding and distant cells via EVs in their microenvironment.

On the other hand, EVs have great potential as cancer biomarkers. Although a tissue biopsy is required for definitive diagnosis of cancers, it is an invasive procedure for patients. Therefore, it is difficult to perform tissue biopsy frequently. In daily clinical practice, rapid, simple, and less invasive biomarkers are required. Recently, liquid biopsy has emerged as an evolutionary technique that provides novel perspectives on cancer treatment $(23,24)$. In particular, EVbased liquid biopsy shows some advantages compared to tissue biopsy. First, EVs have been repeatedly reported to be stable in almost all types of body fluids, and they are less-invasive to collect (25). Second, EVs reflect the current state of the disease in a timely manner by inheriting specific biomolecules from the parental cells. For these reasons, EVbased liquid biopsy allows us to monitor the disease with multiple minimally invasive snapshots. In the next section, we summarize the recent studies of EVs in BCa and discuss their clinical application. 


\section{The potential of urinary EVs in BCa}

As $\mathrm{BCa}$ is bathed in urine, most $\mathrm{EV}$ biomarker studies for $\mathrm{BCa}$ use urine specimens. On the other hand, some studies have shown that $\mathrm{EV}$ s from $\mathrm{BCa}$ are also released into systemic circulation $(26,27)$. Although whether urine or blood is the better source of BCa biomarkers remains to be answered, urine can contain larger amounts of $\mathrm{EVs}$ from $\mathrm{BCa}$ than blood, and most studies are focused on urinary biomarkers. Thus, in this review, we focused on the potential of EVs in urine specimens as biomarkers for the diagnosis and follow-up of patients with $\mathrm{BCa}$. Among the contents of urinary EVs, noncoding RNAs, mRNAs, and proteins are frequently reported as biomarkers for BCa.

\section{miRNA}

miRNAs are small noncoding RNAs of 20-25 nucleotides in length that post-transcriptionally regulate the expression of thousands of genes, affecting various kinds of biological processes (28). miRNAs can be bound to RNA-binding proteins, including Argonaute 2 protein, or packed in EVs and are highly stable in various kinds of body fluids. They can not only exert decisive roles in cell-to-cell communication but also have the potential to serve as biomarkers for various kinds of cancers (29). To date, the majority of the studies of urinary EVs have focused on miRNAs.

First, Armstrong et al. investigated the miRNA molecular profile of matched tumour tissue and body fluids, including urinary EVs, from 16 patients with $\mathrm{BCa}$ and found that miR-205, miR-2003c-3p, and miR-29b-3p were common to tumour tissue and urinary EVs (30). As muscle invasive $\mathrm{BCa}$ (MIBC) is characterized by specific molecular alterations (31), it was expected that miRNAs in urinary EVs also reflected the specific characteristics of MIBC. Baumgart et al. selected 9 candidate miRNAs that were deregulated in both invasive $\mathrm{BCa}$ cell lines and their secreted EVs compared to non-invasive cells and their EVs (32). However, they could not validate the results of these miRNAs using patients with MIBC and non-MIBC (NMIBC). Therefore, further research is needed to identify clinically important miRNAs in urinary EVs to detect MIBC.

Andreu et al. and Matsuzaki et al. directly investigated miRNAs in EVs. Andreu et al. used microarray analysis of miRNAs to select candidate miRNAs in urinary EVs from 4 patients with high-grade $\mathrm{BCa}$ and 4 healthy nonsmokers. Subsequently, selected miRNAs were validated in the different cohorts (9 healthy non-smokers, 16 patients with low-grade $\mathrm{BCa}$, and 18 patients with high-grade $\mathrm{BCa}$ ). The results showed that miR-375 could identify highgrade $\mathrm{BCa}$, while miR-146a could be used as a biomarker for low-grade $\mathrm{BCa}$ (33). In another study, Matsuzaki et al. performed microarray analysis of miRNAs of urinary EVs from 6 patients with $\mathrm{BCa}$ and 3 healthy donors and selected candidate miRNAs that were enriched in EVs from patients with $\mathrm{BCa}$. They subsequently validated the expression of a miRNA-independent cohort $(n=60)$ and showed significantly higher levels of miR-21-5p in BCa patients (34). As the tumour-surrounding cells can also secrete specific miRNAs in urinary EVs, it might be reasonable to directly investigate urinary EVs without selecting miRNAs on the basis of the results of tumour cells.

\section{Other non-coding RNAs}

To date, two kinds of non-coding RNAs, long non-coding RNAs (lncRNAs) and circular RNAs (circRNAs), have been reported to serve as biomarkers in urinary EVs for $\mathrm{BCa}$ (35-43). LncRNAs are composed of more than 200 nucleotides. Dysregulation of IncRNA expression has been reported to play district roles in malignant processes, such as tumour progression or development of metastasis $(35,36)$. Recently, IncRNAs in urinary EVs have been isolated as potential biomarkers. Berrondo et al. reported that lncRNAs, HOX transcript antisense RNA (HOTAIR), HOX-AS-2, ANRIL, and linc-ROR, were enriched in urinary EVs from $\mathrm{BCa}$ patients with high-grade muscleinvasive disease $(\mathrm{n}=8)$ compared to healthy volunteers $(\mathrm{n}=5)$ (37). In the same study, they also identified additional and novel lncRNAs (HYMA1, LINC0047, LOC100506688, and OTX2-AS1) in urinary EVs, which were upregulated in invasive $\mathrm{BCa}(\mathrm{n}=8)$ compared to healthy volunteers $(\mathrm{n}=3)$, using RNA sequences (37). Zhan et al. screened and evaluated the expression of 8 lncRNAs that have been reported to play functional roles in tumorigenesis in a training set and validation set using qRT-PCR. They established a panel of three lncRNAs (MALAT1, PCAT-1, and SPRY4-IT1) for BCa diagnosis in the training set (104 BCa patients and 104 healthy volunteers) and subsequently validated its performance in the validation set $(80 \mathrm{BCa}$ patients and 80 healthy volunteers); it had an area under the ROC curve (AUC) of 0.813 (38). In addition, they also revealed that the expression of PCAT- 1 could act as a biomarker to predict the recurrence of $\mathrm{BCa}$ (38).

CircRNAs are a class of non-coding RNAs with 
covalently closed loop structures that are formed from exon direct back splicing of pre-mRNA transcripts (39). Although circRNAs have been thought of as no more than junk RNA molecules, recent studies have shown that they have multiple regulatory roles $(40,42)$. In BCa patients, circPRMT5 was found to promote epithelial-mesenchymal transformation (EMT) by sponging miR-30c, contributing to tumour progression (43). Additionally, circPRMT5 was upregulated in urinary EVs from 18 patients with $\mathrm{BCa}$ compared to 14 healthy volunteers, and its expression was associated with tumour metastasis (43).

\section{mRNAs}

mRNA is reported to be contained in EVs. mRNAs transferred into recipient cells via EVs can be translated into proteins and provide phenotypical changes (44). Murakami et al. performed RNA-seq analysis on urinary EVs from 12 BCa patients and selected 12 mRNA candidates for the detection of $\mathrm{BCa}$. In the validation cohort $(\mathrm{n}=173)$ using qRT-PCR, SLC2A1, GPRC5, and KRT17 were confirmed as diagnostic markers. These three mRNAs were able to detect both NMIBC and MIBC and outperformed conventional cytology or BCa biomarkers such as BTA (45). Additionally, a panel of the three mRNAs in combination with urine cytology improved the detection of pT1 and higher stage BCa compared to that with urine cytology alone (AUC 0.93). Furthermore, they revealed that the panel of the three genes could be useful for assessing patients with negative cytology results (45). On the other hand, Perez et al. screened urinary EVs using an mRNA microarray $(\mathrm{n}=11)$ and validated that LASS2 and GALNT1 were only detected in urinary EVs from BCa patients, while ARHGEF39 and FOXO3 were only detected in urinary EVs from non-cancer controls using PCR $(n=4)(46)$. These genes were not detected or differentially expressed in the RNA-seq data from the study of Murakami et al. (45). The differences in these findings may have resulted from the limited number of samples used in RNA-seq or microarrays; therefore, further research will be necessary to evaluate these results using a large number of samples.

\section{Proteins}

Proteins are one of the most investigated molecules in EV biomarker studies. Based on proteomic analysis, several reports have shown the usefulness of proteins in urinary EVs. However, the proteins identified by proteomic analysis differ among studies (47-49). On the other hand, other studies focused on specific proteins in urinary EVs and evaluated their diagnostic or prognostic value in $\mathrm{BCa}$ management (50-54). Lin et al. developed matrix-assisted laser desorption ionization time-of flight (MALDI-TOF) platform and enrolled 129 urothelial carcinoma (UC) patients and 62 participants without UC for determining diagnostic markers. In this study, they identified alpha 1 -antitrypsin and $\mathrm{H} 2 \mathrm{~B} 1 \mathrm{~K}$ in urinary EVs, and these proteins could be diagnostic and prognostic biomarkers for BCa. Verification by immunohistochemistry revealed that these proteins were significantly upregulated in $\mathrm{BCa}$ tissue compared with normal tissue (50). Silvers et al. reported that four proteins (HEXB, S100A4, SND1, and TALDO1) were enriched in urinary EVs from patients with BCa compared to healthy controls (51). In another study, Chen et al. proposed that TACSTD2 in urinary EVs has diagnostic value as a biomarker for patients with $\mathrm{BCa}$ (52).

In addition, several reports have focused on the aggressiveness of $\mathrm{BCa}$ and proteins in urinary EVs. Beckham et al. showed that epidermal growth factor-like repeats and discoidin 1-like domain 3 (EDIL-3) in EVs derived from $\mathrm{BCa}$ cells facilitate angiogenesis and promote the migration of urothelial and endothelial cells in vitro. In the same study, EDIL-3 in EVs from urinary EVs from 12 MIBC patients was significantly higher than that in EVs from 12 healthy volunteers, suggesting its effectiveness as a biomarker for MIBC (53). In another study, Silvers et al. found that MIBC cells secrete EVs containing periostin and that the transfer of periostin activates ERK oncogenic signals, promoting cell aggressiveness in recipient cells. They also found that the expression of periostin in urinary EVs was higher in 4 patients with MIBC than in 4 healthy controls and 3 patients with NMIBC following transurethral resection of bladder tumour (TURBT) (54). The different technical approaches and small series of patients are thought to be the main reasons for the heterogeneity of the results between the studies (Table 1).

\section{The current hurdles towards clinical application}

Although urinary EVs appear to be a valuable source for the establishment of novel biomarkers in BCa, there are several points to discuss regarding their clinical application. In this section, we summarize the advantages and disadvantages of EV-associated noncoding RNAs and proteins.

The advantage of targeting noncoding RNAs in EVs is that we can repeatedly and readily evaluate their expression 
Table 1 Potential use of urinary extracellular vesicles in bladder cancer

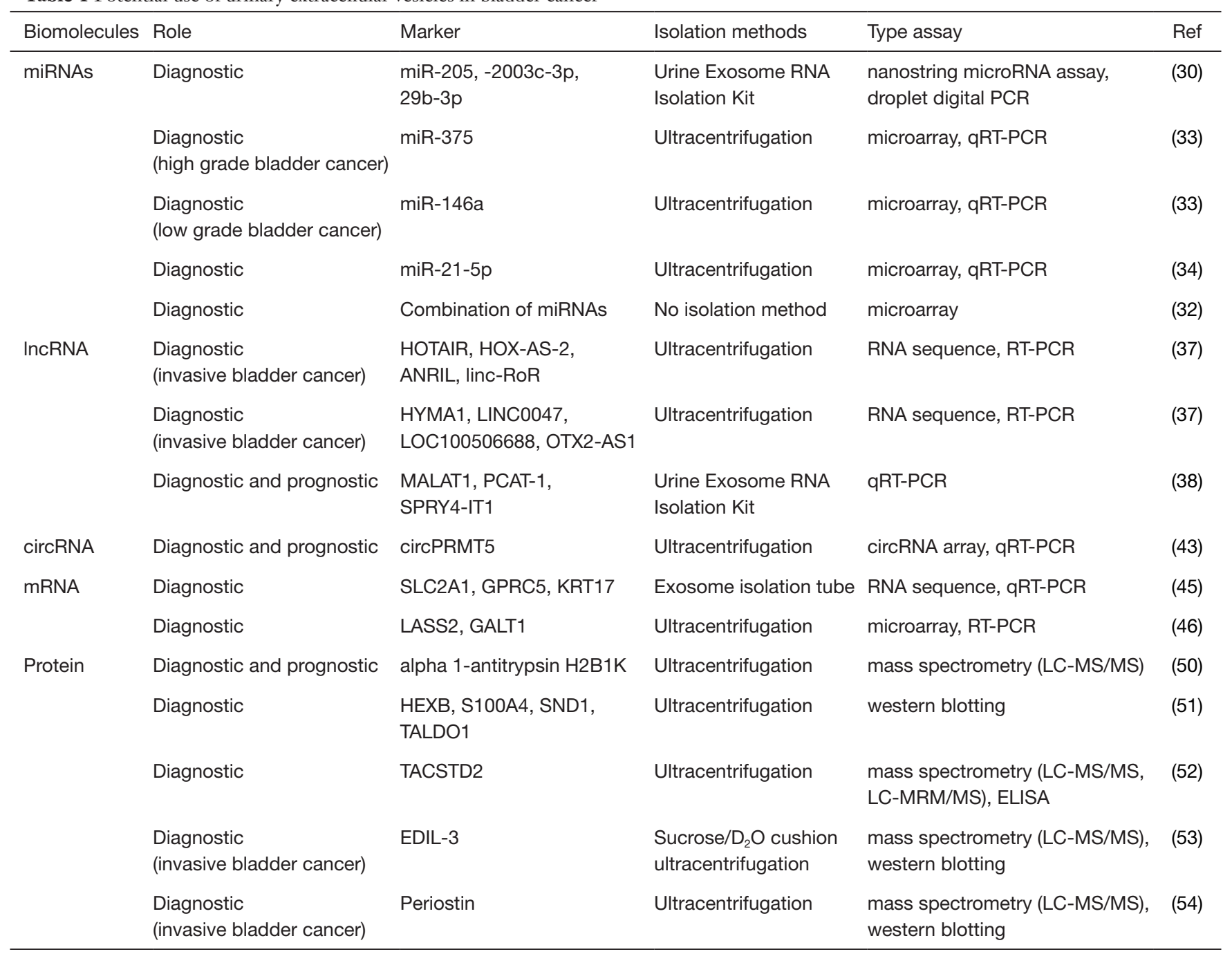

by using real-time PCR with a small number of samples. In addition, as noncoding RNAs in EVs are covered by lipid bilayer membranes, their stability and repeatability are guaranteed. On the other hand, to establish a platform that accurately evaluates the expression level of noncoding RNA, a standardized method to collect EVs is required. To date, ultracentrifugation and density gradient-based isolation are conventional gold standard methods to isolate $\mathrm{EVs}$, but these procedure require a lot of time and effort (55). Commercial EV isolation kits are fast and simple methods; however, they may be a relatively rough isolation procedure with contaminating soluble proteins (56). Recently, to overcome these drawbacks, microfluidics has gained attention. Microfluidic approaches have the potential to revolutionize and realize high-throughput $\mathrm{EV}$ analysis. To date, these approaches have successfully demonstrated the isolation of EVs (57-60). For example, in urinary EVs, Yasui et al. established a nanowire-based method for evaluating urine EV-encapsulated miRNAs (60). This device was able to extract much larger kinds of miRNAs than conventional ultracentrifugation or a commercial $\mathrm{EV}$ isolation kit from a smaller sample volume and over a shorter period of time (60). Therefore, the establishment of novel devices like these to purify EVs is required for the clinical application of urinary $\mathrm{EV}$-associated noncoding RNAs. Moreover, there is no consensus on which small RNA is suitable for use as an internal control, which presents an additional problem. For EV-associated miRNAs, spike-in external controls, such as non-human (Caenorbabditis elegans) miRNAs, are commonly used in normalization. However, it is difficult to 
precisely manage the amount of the external controls added to different samples. The use of unchanged miRNAs as endogenous controls might be ideal, but no consensus has been reached to date (29).

The advantage of focusing on proteins on EVs is that we can directly assess the EVs without $\mathrm{EV}$ isolation steps, such as ultracentrifugation. Ultracentrifugation requires a large amount of time and is not suitable for the clinical setting. Therefore, targeting the proteins on urinary EVs has great potential for establishing feasible biomarkers. To date, several detection methods using antibody-based assays have been used to characterize the proteins expressed on EVs (61-66). For instance, Yoshioka et al. established a method called the ExoScreen assay, which can detect circulating EVs in serum based on an amplified luminescent proximity homogeneous assay using photosensitizer beads and two specific antigens residing on EVs. In this study, they revealed that the ExoScreen assay detected colorectal cancer more exactly than the conventional tumour marker CA19-9 (61). However, these detection methods were examined in a relatively small number of patients. In addition, as the proteins on EVs are sensitive to collection and preservation conditions $(67,68)$, the examination should be performed under strict protocols. Furthermore, the expression of protein on EVs in urine can be affected by urinary concentration. Urinary concentration can be easily influenced by the amount of water intake. Therefore, to normalize this, several studies reported that the creatinine concentration of undiluted urine is highly correlated with the particle counts of EVs in the urine specimen and can be used for normalization in urinary EVs (34,69-71).

In the studies of both urinary EV-associated noncoding RNAs and proteins, the difference in target molecules among the studies can be a hurdle to clinical application. To validate the usefulness of each molecule in urinary EVs, large-scale and inter-laboratory research should be performed. In addition, the combination of these molecules would be valuable for establishing a useful urinary EV panel for BCa management.

Although several issues remain, urinary $\mathrm{EV}$-associated noncoding RNAs and proteins could be novel and useful liquid biopsy tools for the management of BCa.

\section{Points of view from clinicians in BCa management}

There are several topics that can directly influence daily clinical practice in $\mathrm{BCa}$ management. In this section, we highlight recent clinical concerns in $\mathrm{BCa}$ and discuss the potential application of urinary EVs.

While radical cystectomy remains the gold standard strategy for invasive localized $\mathrm{BCa}$ or $\mathrm{BCa}$ with a high risk of dissemination, it is an invasive surgery with a severe impact on the quality of life of patients (72). First, to diagnose MIBC, TURBT is performed in clinical practice. However, a useful biomarker for predicting MIBC prior to TURBT does not exist. In patients with invasive $\mathrm{BCa}$, the tumours easily spread into lymphatic vessels and blood vessels; therefore, the amount of EVs released into systemic circulation will also be increased. Indeed, in gastric cancer, there is a report that the number of plasma EVs was correlated with the stage of cancer development (73). Therefore, in BCa, the amount of circulating EVs from blood samples or the ratio of urinary EVs to EVs in blood might be an important biomarker for predicting invasive $\mathrm{BCa}$.

Recent whole genome characterization of primary $\mathrm{BCa}$ elucidated that it is a heterogeneous disease that can be grouped into several molecular subtypes. This classification advanced our understanding of the biology of $\mathrm{BCa}$ (74). Specific genomic alterations are enriched in particular molecular mutations and copy number aberrations that underline progression patterns and biological and clinical properties. Intriguingly, several studies reported that the responses to chemotherapy and immunotherapy depend on the molecular subtypes of $\mathrm{BCa}$ (75-78). In prostate cancer (PCa), AR-V7, which is the most well-known AR splice variant, is associated with resistance to androgen-receptor axis targeted (ARAT) agents in metastatic castrationresistant PCa (mCRPC) and has the potential to be a good prognostic marker $(79,80)$. Indeed, AR-V7 in plasma EVs is reported to be a biomarker to predict resistance to ARAT agents in patients with mCRPC (81). To date, there is no report to show the relationship between the characteristics of urinary EVs and the molecular subtypes of $\mathrm{BCa}$. However, the contents of urinary EVs can be drastically influenced by primary BCa; therefore, they can also be good candidates for predicting the molecular subtype of $\mathrm{BCa}$.

Until recently, the therapeutic strategy for $\mathrm{BCa}$ had seen little progress. For approximately thirty years, clinicians had the same, limited range of cisplatin-based chemotherapy, such as methotrexate, vinblastine, doxorubicin and cisplatin (MVAC) or gemcitabine-cisplatin (GC), and unfortunately, 5 -year survival rates remained the same $(82,83)$. However, thanks to the introduction of immune checkpoint inhibitor drugs, the overall survival of patients with advanced $\mathrm{BCa}$ is prolonged, and some patients with advanced $\mathrm{BCa}$ who were 
given only months to live are now in durable remission (84). PD-1, which is highly expressed on activated $\mathrm{T}$ cells, and its ligand PD-L1, which is expressed on tumour cells or immune cells, comprise one of the main immune checkpoint pathways that downregulates immune activity in the tumour microenvironment. Blockade of this immune checkpoint pathway has demonstrated clinical activity in several types of solid cancers, including $\mathrm{BCa}(85,86)$. Logically, the expression level of PD-L1 on tumours could be an effective marker to predict the response to immune checkpoint inhibitors in $\mathrm{BCa}$; however, a validated predictive biomarker of response has yet to be defined (86). Therefore, selecting patients who will respond to immune checkpoint inhibitors remains a pressing clinical issue. Another specific issue is that sequential tissue biopsy is an invasive procedure; thus, less-invasive liquid biopsy would be more ideal. To date, several studies have reported that EVs have great potential as biomarkers for immune checkpoint inhibitors. The expression level of PD-L1 on EVs has been demonstrated to be associated with disease progression and immunotherapy response in patients with head and neck squamous cell carcinoma (HNSCC) and melanoma $(87,88)$. In addition, the expression of PDL1 mRNA in EVs has also been reported to be associated with the response to anti-PD-1 antibodies in patients with melanoma and non-small cell lung cancer (NSCLC) (89). Furthermore, recently, the roles of PD-L1 on EVs from cancer cells were reported. Chen et al. revealed that metastatic melanoma cells secrete high levels of PD-L1 on EVs, which bind to PD-1 on CD8 T cells, suppress their functions, and promote tumour progression. In this study, they also revealed that PD-L1 on EVs in patients with melanoma can be a biomarker for predicting the response to anti-PD-1 therapy (90). Poggio et al. revealed that PDL1 on EVs from metastatic PCa cells is a major regulator of tumour progression by suppressing $\mathrm{T}$ cell activation. In the same study, using a PCa syngeneic model, they also showed that resistance to immunotherapy can be caused by PD$\mathrm{L} 1$ on EVs from cancer cells and concluded that PD-L1 on EVs could be a novel target for overcoming resistance to current immunotherapy approaches (91).

\section{Future perspectives for EV-based therapy}

As we mentioned previously, EVs facilitate cell-cell communication to promote tumour development by transferring their contents. Indeed, in $\mathrm{BCa}$, several reports have shown that EVs play key roles in tumour dissemination, e.g., tumorigenesis and progression $(53,92)$, angiogenesis (53), and cancer-associated fibroblasts formation (93). Therefore, inhibition of transmission of EV cargo may provide a potential novel therapeutic strategy for BCa patients.

To date, three strategies of EV-based therapies (inhibition of EV secretion, elimination of circulating EVs, and disruption of EV absorption) have been proposed (21). Among these three strategies, inhibition of EV secretion and elimination of circulating EVs were mainly reported.

Kosaka $e t$ al. reported that the knockdown of neutral sphingomyelinase 2 (nSMase2) reduced EV secretion from breast cancer cells, resulting in the suppression of lung metastasis in vivo (94). However, nSMase2 is also expressed in normal cells (95). Additionally, the downregulation of nSMase2 did not inhibit the secretion of EVs in PCa cells (96); therefore, the suppression of nSMase2 will not provide the same effect on the secretion of EVs in all kinds of cancers. To overcome this dilemma, the mechanism of cancer-specific EV secretion should be investigated. Recently, we established a screening assay to comprehensively screen the regulator genes of $\mathrm{EV}$ secretion, which can be carried out in a short period of time and can be applied to all kinds of cancer $(97,98)$. This highthroughput screening method can contribute to revealing the mechanism involved in EV secretion and contribute to the establishment of EV-based therapy targeting $\mathrm{EV}$ secretion.

Human epidermal growth factor receptor 2 (HER-2) localized on EVs has been shown to interfere with trastuzumab, an anti-HER-2 monoclonal antibody, and contribute to cancer progression (99). Marleau et al. applied this mechanism to establish an EV-targeting strategy for absorbing circulating EVs (100). They developed the hemofiltration system, which can selectively absorb circulating EVs derived from breast cancer cells by targeting HER-2 on the surface of EVs (100). Nishida-Aoki et al. demonstrated that administration of antibodies against $\mathrm{CD} 9$ and $\mathrm{CD} 63$, which are enriched on the surface of $\mathrm{EVs}$, decreased circulating EVs and inhibited cancer progression in an in vivo mouse model (101).

Although there are still many obstacles to overcome before clinical implementation, the reduction of cell-tocell communication via $\mathrm{EVs}$ has great potential as a novel therapeutic strategy in cancers. As the concepts of these strategies can be applied to BCa, EV-based therapies may add additional value to existing therapeutic methods, such as surgery, radiation therapy, and chemotherapy for patients 


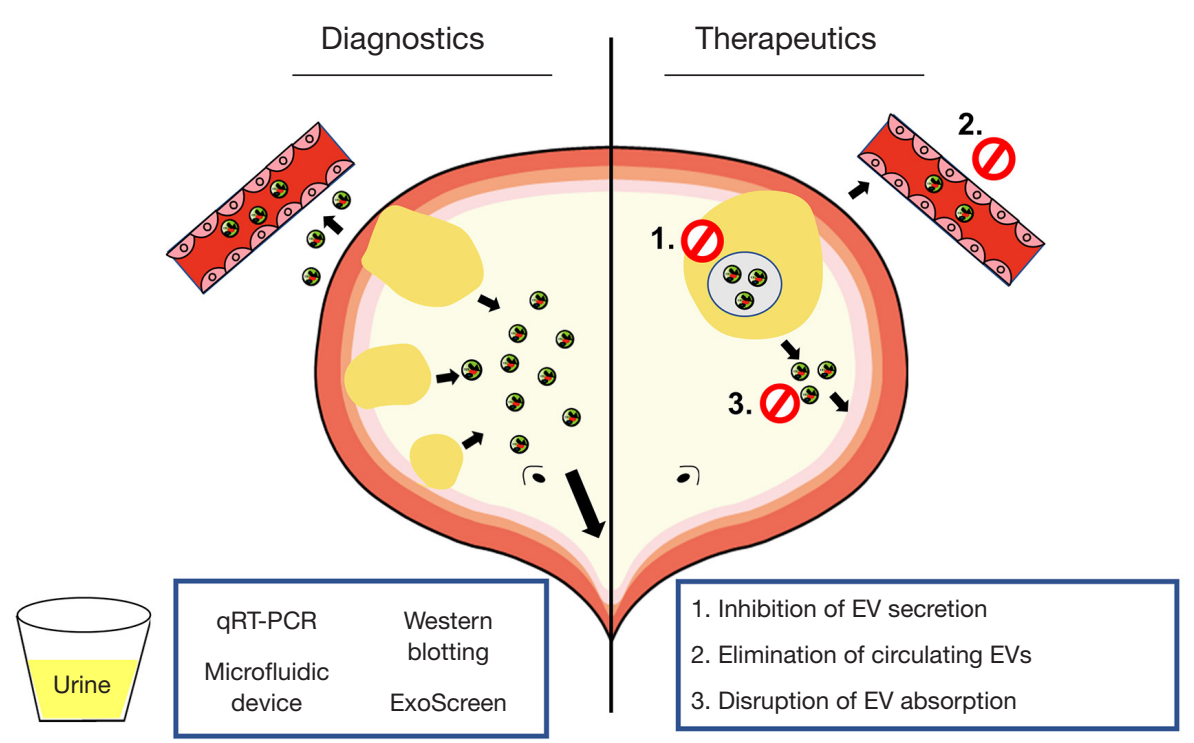

Figure 1 Clinical application of extracellular vesicles (EVs) in bladder cancer management. Urinary EVs are likely to be clinically useful biomarkers for bladder cancer. EV-associated biomolecules can be examined using several modalities, including real-time PCR, western blotting, and ExoScreen assays and microfluidic devices. Three therapeutic applications have been proposed: 1 . inhibition of EV secretion, 2. elimination of circulating EVs, and 3. disruption of EV absorption. These EV-targeted strategies may add additional value to existing therapeutic methods.

with BCa in the near future (Figure 1).

\section{Conclusions}

This review demonstrated the high potential of urinary EVs as biomarkers for patients with BCa. Although none of the EV-based biomarkers has been used in daily clinical practice to date, the speed of $\mathrm{EV}$ research development is astonishing; therefore, novel technologies will overcome the present hurdles. We believe that urinary EVs will be used as clinically useful biomarkers in BCa management.

Additionally, EVs are multifunctional and potent cell-tocell communicators that can contribute to changes in local and distant microenvironments to promote the survival, progression, and invasion of tumour cells. Therefore, EV research has the potential to unveil a novel mechanism of disease progression and to also provide an opportunity for the development of EV-targeted therapeutics. Further research is required; however, $\mathrm{EVs}$ could be used in every aspect of cancer treatment from early diagnosis to treatment of advanced stage cancer patients. We enthusiastically hope that the findings of $\mathrm{EV}$ research will clinically contribute to the treatment of BCa patients.

\section{Acknowledgments}

Funding: This work was supported by the Yamaguchi Endocrine Research Foundation.

\section{Footnote}

Provenance and Peer Review: This article was commissioned by the Guest Editor (Kazutoshi Fujita) for the series "Urinary Biomarkers of Urothelial Malignancies" published in Translational Andrology and Urology. The article has undergone external peer review.

Conflicts of Interest: All authors have completed the ICMJE uniform disclosure form (available at http:// dx.doi.org/10.21037/tau-20-1039). The series "Urinary Biomarkers of Urothelial Malignancies" was commissioned by the editorial office without any funding or sponsorship. The authors have no other conflicts of interest to declare.

Ethical Statement: The authors are accountable for all aspects of the work in ensuring that questions related to the accuracy of integrity of any part of the work are appropriately investigated and resolved. 
Open Access Statement: This is an Open Access article distributed in accordance with the Creative Commons Attribution-NonCommercial-NoDerivs 4.0 International License (CC BY-NC-ND 4.0), which permits the noncommercial replication and distribution of the article with the strict proviso that no changes or edits are made and the original work is properly cited (including links to both the formal publication through the relevant DOI and the license). See: https://creativecommons.org/licenses/by-nc-nd/4.0/.

\section{References}

1. Bray F, Ferlay J, Soerjomataram I, et al. Global cancer statistics 2018: GLOBOCAN estimates of incidence and mortality worldwide for 36 cancers in 185 countries. CA Cancer J Clin 2018;68:394-424.

2. Matsuda T, Okuyama A. Incidence rate for bladder cancer in Japanese in Japan and in the United States from the Cancer Incidence in Five Continents. Jpn J Clin Oncol 2017;47:284-5.

3. Siegel RL, Miller KD, Jemal A. Cancer statistics, 2020. CA Cancer J Clin 2020;70:7-30.

4. Yafi FA, Brimo F, Steinberg J, et al. Prospective analysis of sensitivity and specificity of urinary cytology and other urinary biomarkers for bladder cancer. Urol Oncol 2015;33:66.e25-31.

5. Têtu B. Diagnosis of urothelial carcinoma from urine. Mod Pathol 2009;22 Suppl 2:S53-9.

6. Lotan Y, Roehrborn CG. Sensitivity and specificity of commonly available bladder tumor markers versus cytology: results of a comprehensive literature review and meta-analyses. Urology 2003;61:109-18; discussion 118.

7. Mowatt G, Zhu S, Kilonzo M, et al. Systematic review of the clinical effectiveness and cost-effectiveness of photodynamic diagnosis and urine biomarkers (FISH, ImmunoCyt, NMP22) and cytology for the detection and follow-up of bladder cancer. Health Technol Assess 2010;14:1-331, iii-iv.

8. Burke DM, Shackley DC, O'Reilly PH. The communitybased morbidity of flexible cystoscopy. BJU Int 2002;89:347-9.

9. Biardeau X, Lam O, Ba V, et al. Prospective evaluation of anxiety, pain, and embarrassment associated with cystoscopy and urodynamic testing in clinical practice. Can Urol Assoc J 2017;11:104-10.

10. Schmitz-Dräger BJ, Droller M, Lokeshwar VB, et al. Molecular markers for bladder cancer screening, early diagnosis, and surveillance: the WHO/ICUD consensus.
Urol Int 2015;94:1-24.

11. Hajdinjak T. UroVysion FISH test for detecting urothelial cancers: meta-analysis of diagnostic accuracy and comparison with urinary cytology testing. Urol Oncol 2008;26:646-51.

12. Chang SS, Boorjian SA, Chou R, et al. Diagnosis and Treatment of Non-Muscle Invasive Bladder Cancer: AUA/ SUO Guideline. J Urol 2016;196:1021-9.

13. Fujita Y, Yoshioka Y, Ochiya T. Extracellular vesicle transfer of cancer pathogenic components. Cancer Sci 2016;107:385-90.

14. Urabe F, Kosaka N, Kimura T, et al. Extracellular vesicles: Toward a clinical application in urological cancer treatment. Int J Urol 2018;25:533-43.

15. Raposo G, Stoorvogel W. Extracellular vesicles: exosomes, microvesicles, and friends. J Cell Biol 2013;200:373-83.

16. Colombo M, Raposo G, Théry C. Biogenesis, secretion, and intercellular interactions of exosomes and other extracellular vesicles. Annu Rev Cell Dev Biol 2014;30:255-89.

17. Robbins PD, Morelli AE. Regulation of immune responses by extracellular vesicles. Nat Rev Immunol 2014;14:195-208.

18. Cocucci E, Meldolesi J. Ectosomes and exosomes: shedding the confusion between extracellular vesicles. Trends Cell Biol 2015;25:364-72.

19. Muralidharan-Chari V, Clancy JW, Sedgwick A, et al. Microvesicles: mediators of extracellular communication during cancer progression. J Cell Sci 2010;123:1603-11.

20. Gould SJ, Raposo G. As we wait: coping with an imperfect nomenclature for extracellular vesicles. J Extracell Vesicles 2013. doi: 10.3402/jev.v2i0.20389.

21. Kosaka N, Yoshioka Y, Fujita Y, et al. Versatile roles of extracellular vesicles in cancer. J Clin Invest 2016;126:1163-72.

22. Becker A, Thakur BK, Weiss JM, et al. Extracellular Vesicles in Cancer: Cell-to-Cell Mediators of Metastasis. Cancer cell 2016;30:836-48.

23. Mathai RA, Vidya RVS, Reddy BS, et al. Potential Utility of Liquid Biopsy as a Diagnostic and Prognostic Tool for the Assessment of Solid Tumors: Implications in the Precision Oncology. J Clin Med 2019;8:373.

24. Rossi G, Ignatiadis M. Promises and Pitfalls of Using Liquid Biopsy for Precision Medicine. Cancer Res 2019;79:2798-804.

25. Urabe F, Kosaka N, Ito K, et al. Extracellular vesicles as biomarkers and therapeutic targets for cancer. Am J Physiol Cell Physiol 2020;318:C29-39. 
26. Xue M, Chen W, Xiang A, et al. Hypoxic exosomes facilitate bladder tumor growth and development through transferring long non-coding RNA-UCA1. Mol Cancer 2017;16:143

27. Zhang S, Du L, Wang L, et al. Evaluation of serum exosomal LncRNA-based biomarker panel for diagnosis and recurrence prediction of bladder cancer. J Cell Mol Med 2019;23:1396-405.

28. Pritchard CC, Cheng HH, Tewari M. MicroRNA profiling: approaches and considerations. Nat Rev Genet 2012;13:358-69.

29. Urabe F, Kosaka N, Yoshioka Y, et al. The small vesicular culprits: the investigation of extracellular vesicles as new targets for cancer treatment. Clin Transl Med 2017;6:45.

30. Armstrong DA, Green BB, Seigne JD, et al. MicroRNA molecular profiling from matched tumor and bio-fluids in bladder cancer. Mol Cancer 2015;14:194.

31. Catto JW, Alcaraz A, Bjartell AS, et al. MicroRNA in prostate, bladder, and kidney cancer: a systematic review. Eur Urol 2011;59:671-81.

32. Baumgart S, Hölters S, Ohlmann CH, et al. Exosomes of invasive urothelial carcinoma cells are characterized by a specific miRNA expression signature. Oncotarget 2017;8:58278-91.

33. Andreu Z, Otta Oshiro R, Redruello A, et al. Extracellular vesicles as a source for non-invasive biomarkers in bladder cancer progression.Eur J Pharm Sci 2017;98:70-9.

34. Matsuzaki K, Fujita K, Jingushi K, et al. MiR-21-5p in urinary extracellular vesicles is a novel biomarker of urothelial carcinoma. Oncotarget 2017;8:24668-78.

35. Shen H, Wong LM, Li WT, et al. The Landscape of Long Non-Coding RNA Dysregulation and Clinical Relevance in Muscle Invasive Bladder Urothelial Carcinoma. Cancers (Basel) 2019;11:1919.

36. Gupta RA, Shah N, Wang KC, et al. Long non-coding RNA HOTAIR reprograms chromatin state to promote cancer metastasis. Nature 2010;464:1071-6.

37. Berrondo C, Flax J, Kucherov V, et al. Expression of the Long Non-Coding RNA HOTAIR Correlates with Disease Progression in Bladder Cancer and Is Contained in Bladder Cancer Patient Urinary Exosomes. PloS One 2016;11:e0147236.

38. Zhan Y, Du L, Wang L, et al. Expression signatures of exosomal long non-coding RNAs in urine serve as novel non-invasive biomarkers for diagnosis and recurrence prediction of bladder cancer. Mol Cancer 2018;17:142.

39. Xiao MS, Ai Y, Wilusz JE. Biogenesis and Functions of Circular RNAs Come into Focus. Trends Cell Biol
2020;30:226-40.

40. Yang Y, Gao X, Zhang M, et al. Novel Role of FBXW7 Circular RNA in Repressing Glioma Tumorigenesis. J Natl Cancer Inst 2018;110:304-15.

41. Qiu M, Xia W, Chen R, et al. The Circular RNA circPRKCI Promotes Tumor Growth in Lung Adenocarcinoma. Cancer Res 2018;78:2839-51.

42. Hansen TB, Kjems J, Damgaard CK. Circular RNA and miR-7 in cancer. Cancer Res 2013;73:5609-12.

43. Chen X, Chen RX, Wei WS, et al. PRMT5 Circular RNA Promotes Metastasis of Urothelial Carcinoma of the Bladder through Sponging miR-30c to Induce Epithelial-Mesenchymal Transition. Clin Cancer Res 2018;24:6319-30.

44. Yokoi A, Yoshioka Y, Yamamoto Y, et al. Malignant extracellular vesicles carrying MMP1 mRNA facilitate peritoneal dissemination in ovarian cancer. Nat Commun 2017;8:14470.

45. Murakami T, Yamamoto CM, Akino T, et al. Bladder cancer detection by urinary extracellular vesicle mRNA analysis. Oncotarget 2018;9:32810-21.

46. Perez A, Loizaga A, Arceo R, et al. A Pilot Study on the Potential of RNA-Associated to Urinary Vesicles as a Suitable Non-Invasive Source for Diagnostic Purposes in Bladder Cancer. Cancers 2014;6:179-92.

47. Smalley DM, Sheman NE, Nelson K, et al. Isolation and identification of potential urinary microparticle biomarkers of bladder cancer. J Proteome Res 2008;7:2088-96.

48. Welton JL, Brennan P, Gurney M, et al. Proteomics analysis of vesicles isolated from plasma and urine of prostate cancer patients using a multiplex, aptamer-based protein array. J Extracell Vesicles 2016;5:31209.

49. Lee J, McKinney KQ, Pavlopoulos AJ, et al. Altered Proteome of Extracellular Vesicles Derived from Bladder Cancer Patients Urine. Mol Cells 2018;41:179-87.

50. Lin SY, Chang CH, Wu HC, et al. Proteome Profiling of Urinary Exosomes Identifies Alpha 1-Antitrypsin and $\mathrm{H} 2 \mathrm{~B} 1 \mathrm{~K}$ as Diagnostic and Prognostic Biomarkers for Urothelial Carcinoma. Sci Rep 2016;6:34446.

51. Silvers CR, Miyamoto H, Messing EM, et al. Characterization of urinary extracellular vesicle proteins in muscle-invasive bladder cancer. Oncotarget 2017;8:91199-208.

52. Chen CL, Lai YF, Tang P, et al. Comparative and targeted proteomic analyses of urinary microparticles from bladder cancer and hernia patients. J Proteome Res 2012;11:5611-29.

53. Beckham CJ, Olsen J, Yin PN, et al. Bladder cancer 
exosomes contain EDIL-3/Del1 and facilitate cancer progression. J Urol 2014;192:583-92.

54. Silvers CR, Liu YR, Wu CH, et al. Identification of extracellular vesicle-borne periostin as a feature of muscleinvasive bladder cancer. Oncotarget 2016;7:23335-45.

55. Thind A, Wilson C. Exosomal miRNAs as cancer biomarkers and therapeutic targets. J Extracell Vesicles 2016;5:31292.

56. Van Deun J, Mestdagh P, Sormunen R, et al. The impact of disparate isolation methods for extracellular vesicles on downstream RNA profiling. J Extracell Vesicles 2014. doi: 10.3402/jev.v3.24858.

57. Wang Z, Wu HJ, Fine D, et al. Ciliated micropillars for the microfluidic-based isolation of nanoscale lipid vesicles. Lab Chip 2013;13:2879-82.

58. Lee K, Shao H, Weissleder R, et al. Acoustic purification of extracellular microvesicles. ACS nano 2015;9:2321-7.

59. Liu C, Guo J, Tian F, et al. Field-Free Isolation of Exosomes from Extracellular Vesicles by Microfluidic Viscoelastic Flows. ACS Nano 2017;11:6968-76.

60. Yasui T, Yanagida T, Ito S, et al. Unveiling massive numbers of cancer-related urinary-microRNA candidates via nanowires. Sci Adv 2017;3:e1701133.

61. Yoshioka Y, Kosaka N, Konishi Y, et al. Ultra-sensitive liquid biopsy of circulating extracellular vesicles using ExoScreen. Nat commun 2014;5:3591.

62. Jørgensen M, Bæk R, Pedersen S, et al. Extracellular Vesicle (EV) Array: microarray capturing of exosomes and other extracellular vesicles for multiplexed phenotyping. J Extracell Vesicles 2013. doi: 10.3402/jev.v2i0.20920.

63. Shao H, Chung J, Balaj L, et al. Protein typing of circulating microvesicles allows real-time monitoring of glioblastoma therapy. Nat Med 2012;18:1835-40.

64. Im H, Shao H, Park YI, et al. Label-free detection and molecular profiling of exosomes with a nano-plasmonic sensor. Nat Biotechnol 2014;32:490-5.

65. Zhao Z, Yang Y, Zeng Y, et al. A microfluidic ExoSearch chip for multiplexed exosome detection towards bloodbased ovarian cancer diagnosis. Lab Chip 2016;16:489-96.

66. Liang K, Liu F, Fan J, et al. Nanoplasmonic Quantification of Tumor-derived Extracellular Vesicles in Plasma Microsamples for Diagnosis and Treatment Monitoring. Nat Biomed Eng 2017;1:0021.

67. Jeyaram A, Jay SM. Preservation and Storage Stability of Extracellular Vesicles for Therapeutic Applications. AAPS J 2017;20:1.

68. Maroto R, Zhao Y, Jamaluddin M, et al. Effects of storage temperature on airway exosome integrity for diagnostic and functional analyses. J Extracell Vesicles 2017;6:1359478.

69. Zhou H, Yuen PS, Pisitkun T, et al. Collection, storage, preservation, and normalization of human urinary exosomes for biomarker discovery. Kidney int 2006;69:1471-6.

70. Esteva-Font C, Wang X, Ars E, et al. Are sodium transporters in urinary exosomes reliable markers of tubular sodium reabsorption in hypertensive patients? Nephron Physiol 2010;114:p25-34.

71. Turco AE, Lam W, Rule AD, et al. Specific renal parenchymal-derived urinary extracellular vesicles identify age-associated structural changes in living donor kidneys. J Extracell Vesicles 2016;5:29642.

72. Porter MP, Penson DF. Health related quality of life after radical cystectomy and urinary diversion for bladder cancer: a systematic review and critical analysis of the literature. J Urol 2005;173:1318-22.

73. Baran J, Baj-Krzyworzeka M, Weglarczyk K, et al. Circulating tumour-derived microvesicles in plasma of gastric cancer patients. Cancer Immunol Immunother 2010;59:841-50.

74. Robertson AG, Kim J, Al-Ahmadie H, et al. Comprehensive Molecular Characterization of MuscleInvasive Bladder Cancer. Cell 2017;171:540-56.e25.

75. Rosenberg JE, Hoffman-Censits J, Powles T, et al. Atezolizumab in patients with locally advanced and metastatic urothelial carcinoma who have progressed following treatment with platinum-based chemotherapy: a single-arm, multicentre, phase 2 trial. Lancet 2016;387:1909-20.

76. Necchi A, Raggi D, Gallina A, et al. Impact of Molecular Subtyping and Immune Infiltration on Pathological Response and Outcome Following Neoadjuvant Pembrolizumab in Muscle-invasive Bladder Cancer. Eur Urol 2020;77:701-10.

77. Mariathasan S, Turley SJ, Nickles D, et al. TGF $\beta$ attenuates tumour response to PD-L1 blockade by contributing to exclusion of T cells. Nature 2018;554:544-8.

78. Kamoun A, de Reyniès A, Allory Y, et al. A Consensus Molecular Classification of Muscle-invasive Bladder Cancer. Eur Urol 2020;77:420-33.

79. Antonarakis ES, Lu C, Wang H, et al. AR-V7 and resistance to enzalutamide and abiraterone in prostate cancer. N Engl J Med 2014;371:1028-38.

80. Seitz AK, Thoene S, Bietenbeck A, et al. AR-V7 in Peripheral Whole Blood of Patients with Castration- 
resistant Prostate Cancer: Association with Treatmentspecific Outcome Under Abiraterone and Enzalutamide. Eur Urol 2017;72:828-34.

81. Del Re M, Biasco E, Crucitta S, et al. The Detection of Androgen Receptor Splice Variant 7 in Plasma-derived Exosomal RNA Strongly Predicts Resistance to Hormonal Therapy in Metastatic Prostate Cancer Patients. Eur Urol 2017;71:680-7.

82. Bellmunt J, Orsola A, Leow JJ, et al. Bladder cancer: ESMO Practice Guidelines for diagnosis, treatment and follow-up. Ann Oncol 2014;25 Suppl 3:iii40-8.

83. Berdik C. Unlocking bladder cancer. Nature 2017;551:S34-5.

84. Abdelhakam D, Young PR, Jain MK, et al. Complete remission with immunotherapy: Case report of a patient with metastatic bladder cancer to the humerus. Urol Case Rep 2020;30:101130.

85. Brahmer JR, Tykodi SS, Chow LQ, et al. Safety and activity of anti-PD-L1 antibody in patients with advanced cancer. N Engl J Med 2012;366:2455-65.

86. Hussain SA, Birtle A, Crabb S, et al. From Clinical Trials to Real-life Clinical Practice: The Role of Immunotherapy with PD-1/PD-L1 Inhibitors in Advanced Urothelial Carcinoma. Eur Urol Oncol 2018;1:486-500.

87. Theodoraki MN, Yerneni SS, Hoffmann TK, et al. Clinical Significance of PD-L1(+) Exosomes in Plasma of Head and Neck Cancer Patients. Clin Cancer Res 2018;24:896-905.

88. Cordonnier M, Nardin C, Chanteloup G, et al. Tracking the evolution of circulating exosomal-PD-L1 to monitor melanoma patients. J Extracell Vesicles 2020;9:1710899.

89. Del Re M, Marconcini R, Pasquini G, et al. PD-L1 mRNA expression in plasma-derived exosomes is associated with response to anti-PD-1 antibodies in melanoma and NSCLC. Br J Cancer 2018;118:820-4.

90. Chen G, Huang AC, Zhang W, et al. Exosomal PD-L1 contributes to immunosuppression and is associated with anti-PD-1 response. Nature 2018;560:382-6.

Cite this article as: Urabe F, Kimura T, Ito K, Yamamoto Y, Tsuzuki S, Miki J, Ochiya T, Egawa S. Urinary extracellular vesicles: a rising star in bladder cancer management. Transl Androl Urol 2021;10(4):1878-1889. doi: 10.21037/tau-20-1039
91. Poggio M, Hu T, Pai CC, et al. Suppression of Exosomal PD-L1 Induces Systemic Anti-tumor Immunity and Memory. Cell 2019;177:414-27.e13.

92. Franzen CA, Blackwell RH, Todorovic V, et al. Urothelial cells undergo epithelial-to-mesenchymal transition after exposure to muscle invasive bladder cancer exosomes. Oncogenesis 2015;4:e163.

93. Goulet CR, Champagne A, Bernard G, et al. Cancerassociated fibroblasts induce epithelial-mesenchymal transition of bladder cancer cells through paracrine IL-6 signalling. BMC Cancer 2019;19:137.

94. Kosaka N, Iguchi H, Hagiwara K, et al. Neutral sphingomyelinase 2 (nSMase2)-dependent exosomal transfer of angiogenic microRNAs regulate cancer cell metastasis. J Biol Chem 2013;288:10849-59.

95. Yuyama K, Sun H, Mitsutake S, et al. Sphingolipidmodulated exosome secretion promotes clearance of amyloid- $\beta$ by microglia. J Biol Chem 2012;287:10977-89.

96. Phuyal S, Hessvik NP, Skotland T, et al. Regulation of exosome release by glycosphingolipids and flotillins. FEBS J 2014;281:2214-27.

97. Urabe F, Kosaka N, Sawa Y, et al. miR-26a regulates extracellular vesicle secretion from prostate cancer cells via targeting SHC4, PFDN4, and CHORDC1. Sci Adv 2020;6:eaay3051.

98. Urabe F, Kosaka N, Sawa Y, et al. The miR-1908/SRM regulatory axis contributes to extracellular vesicle secretion in prostate cancer. Cancer sci 2020;111:3258-67.

99. Ciravolo V, Huber V, Ghedini GC, et al. Potential role of HER2-overexpressing exosomes in countering trastuzumabbased therapy. J Cell Physiol 2012;227:658-67.

100. Marleau AM, Chen CS, Joyce JA, et al. Exosome removal as a therapeutic adjuvant in cancer. J Transl Med 2012;10:134.

101. Nishida-Aoki N, Tominaga N, Takeshita F, et al. Disruption of Circulating Extracellular Vesicles as a Novel Therapeutic Strategy against Cancer Metastasis. Mol Ther 2017;25:181-91. 